\title{
Implante subcutáneo de un carcinoma hepatocelular tras la punción aspiración con aguja fina
}

\author{
D. Martínez Ramos, C. Villegas Cánovas, V. Senent Vizcaíno, C. Rodríguez Pereira ${ }^{1}$, J. Escrig Sos, \\ V. Ángel Yepes y J. L. Salvador-Sanchís \\ Servicios de Cirugía General y del Aparato Digestivo y 'Anatomía Patológica. Hospital General de Castellón
}

\begin{abstract}
RESUMEN
Los implantes subcutáneos son una complicación rara tras la punción aspiración con aguja fina de los carcinomas hepatocelulares. Los autores describen un caso de implante subcutáneo neoplásico en una mujer de 70 años con cirrosis hepática por virus $\mathrm{C}$ complicada con un carcinoma hepatocelular. Se efectuó una punción aspiración con aguja fina en el segmento II hepático. El implante tumoral se desarrolló en el trayecto de la punción aspiración. La tumoración subcutánea fue extirpada quirúrgicamente y el estudio anatomopatológico confirmó que se trataba de un carcinoma hepatocelular bien diferenciado.
\end{abstract}

Palabras clave: Implante subcutáneo. Metástasis. Carcinoma hepatocelular. Punción aspiración aguja fina.

\begin{abstract}
Subcutaneous tumor seeding after fine-needle percutaneous biopsy for hepatocellular carcinoma is a rarely seen complication. The authors describe a case of subcutaneous neoplastic seeding in a 70-year-old woman with chronic hepatitis $\mathrm{C}$ virus complicated by hepatocellular carcinoma. Ultrasonically guided fine-needle aspiration biopsy was performed in segment II of the liver. The neoplastic seeding developed along the needle track used to carry out the fine-needle biopsy. The subcutaneous tumor was excised, and histological examination revealed a well-differentiated hepatocellular carcinoma.
\end{abstract}

Key words: Subcutaneous seeding. Metastasis. Hepatocellular carcinoma. Fine-needle biopsy.

Martínez Ramos D, Villegas Cánovas C, Senent Vizcaíno V, Rodríguez Pereira C, Escrig Sos J, Ángel Yepes V, Salvador-Sanchís JL. Implante subcutáneo de un carcinoma hepatocelular tras la punción aspiración con aguja fina. Rev Esp Enferm Dig 2007; 99: 354357.

\section{INTRODUCCIÓN}

La biopsia hepática se considera la técnica más sensible y específica para determinar la naturaleza y la gravedad de cualquier lesión hepática, existiendo cuatro grandes formas de obtener tejido hepático para su estudio anatomopatológico: biopsia percutánea, biopsia transyugular, biopsia laparoscópica y punción aspiración con aguja fina (1).

La punción aspiración con aguja fina (PAAF), guiada por ecografía o por tomografía axial computadorizada (TC), ha

Recibido: 08-05-06

Aceptado: 13-12-06.

Correspondencia: David Martínez Ramos. Servicio de Cirugía General y del Aparato Digestivo. Hospital General de Castellón. Avda Benicàssim, s/n.12004 Castellón.e-mail: davidmartinez@comcas.es demostrado ser una técnica segura y eficaz en el diagnóstico de lesiones focales hepáticas, con escasas complicaciones y una alta rentabilidad diagnóstica. Sin embargo, aunque de forma muy esporádica, algunos autores han comunicado la implantación y proliferación de células neoplásicas en el trayecto de la punción de lesiones tumorales malignas. Se trata de una complicación muy infrecuente, de la que únicamente se han podido publicar casos clínicos aislados, por lo que el conocimiento de un nuevo caso, como el presentado, puede ser de gran interés.

\section{CASO CLÍNICO}

Mujer de 70 años, remitida a nuestra consulta por presentar una tumoración nodular, móvil, violácea, de consistencia dura, de límites bien definidos, de $4 \mathrm{~cm}$ de diámetro máximo, localizada en región subxifoidea (Fig. 1). La le- 


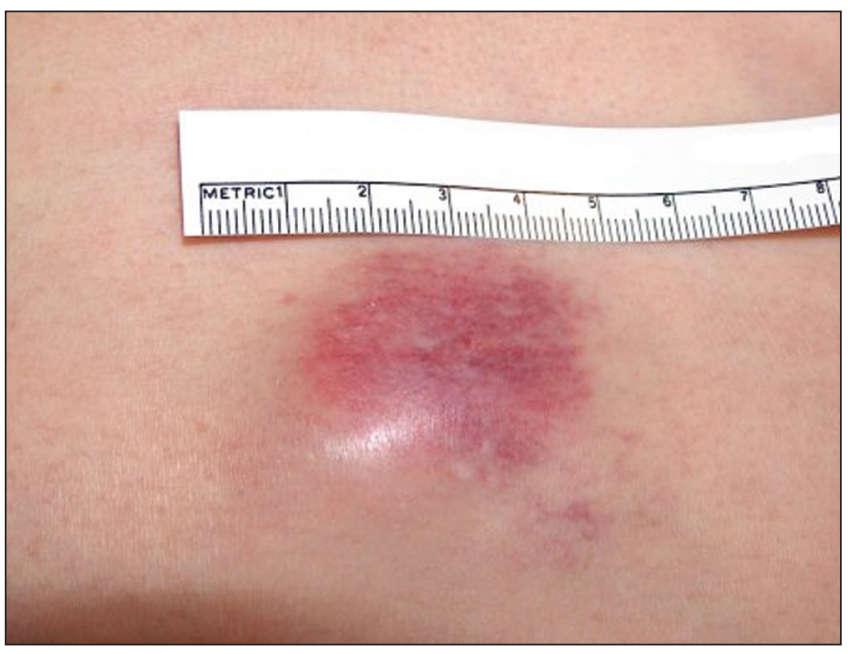

Fig. 1. Aspecto clínico de la tumoración.

sión había aparecido como una pequeña pápula rosada 6 meses antes y su crecimiento había sido rápido y progresivo, hasta llegar a ser ligeramente doloroso. Como antecedente de interés presentaba un carcinoma hepatocelular difuso con un nódulo de $5 \mathrm{~cm}$ en el lóbulo hepático izquierdo y múltiples nódulos en el lóbulo hepático derecho, todo ello sobre una cirrosis hepática por virus de la hepatitis C. El carcinoma había sido diagnosticado 15 meses antes mediante pruebas de imagen (TC y resonancia magnética), $\alpha$ fetoproteína $>300 \mathrm{ng} / \mathrm{mL}$ e histología (PAAF y tru-cut). La extensión y el tamaño tumoral contraindicaron la intervención quirúrgica, por lo que se consideró el tratamiento con quimioembolizaciones supraselectivas, mediante abordaje por arteria femoral común derecha, utilizando lipiodol, cisplatino y embosferas de 300 a 500 micras de diámetro. En los análisis de laboratorio actuales destacaba: LDH 482 UI/L, GOT 182 UI/L, GPT 166 UI/L, $\alpha$-fetoproteína de $324,5 \mathrm{ng} / \mathrm{mL}$. Se realizó una TC abdominal en la que se apreció una lesión sólida de $3,5 \mathrm{~cm}$ localizada en tejido celular subcutáneo, sin continuidad intraabdominal, de límites bien definidos, isodensa con el hígado, que no se apreciaba en controles previos, sugestiva de implante tumoral (Fig. 2). Asimismo, se visualizaron las múltiples lesiones en los segmentos II, III, IV y VII conocidas previamente. La localización de la lesión subcutánea coincidía con el trayecto de punción para la biopsia del segmento II realizada meses antes. No se demostró enfermedad metastásica en ningún otro lugar.

Con el diagnóstico de metástasis subcutánea de un carcinoma hepatocelular se indicó cirugía exerética de la lesión, que se efectuó bajo anestesia general sin complicaciones.

Macroscópicamente, la pieza quirúrgica consistió en una elipse cutánea con abundante tejido subcutáneo, que contenía una tumoración sobreelevada, no ulcerada, que ocupaba un área de $5 \times 2,5 \times 2 \mathrm{~cm}$, de consistencia carnosa al corte, pardo-amarillenta, con zonas hemorrágicas, bien delimitada pero no encapsulada, situada inmediatamente por debajo de la piel (Fig. 3a). Microscópicamente

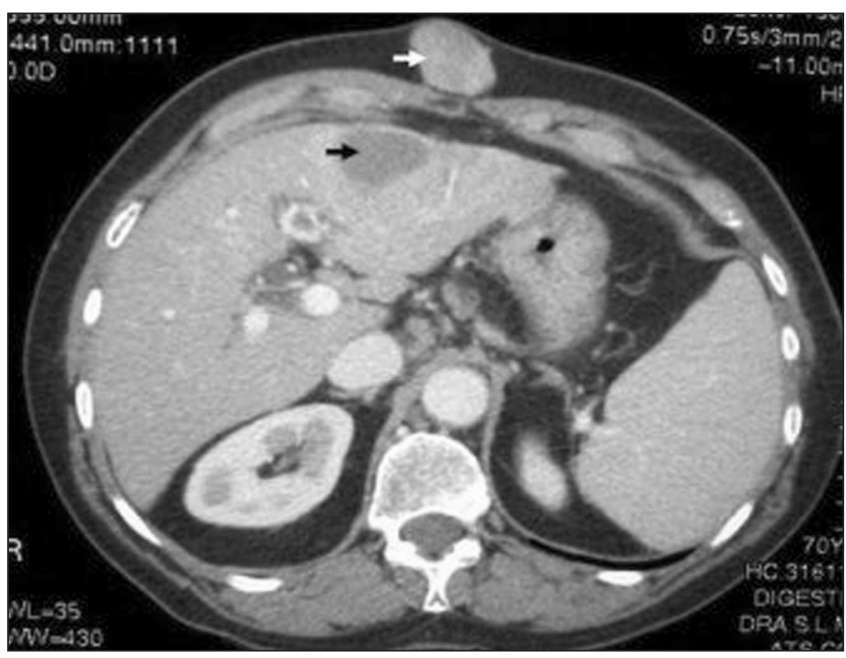

Fig. 2. TC abdominal en la que se observa el implante subcutáneo (flecha blanca) y la lesión tumoral hepática en el segmento II, correspondiente al carcinoma hepatocelular biopsiado meses antes (flecha negra). Nótese la alineación entre la lesión hepática, el implante cutáneo y la zona de punción subxifoidea, correspondiendo al trayecto de la PAAF.

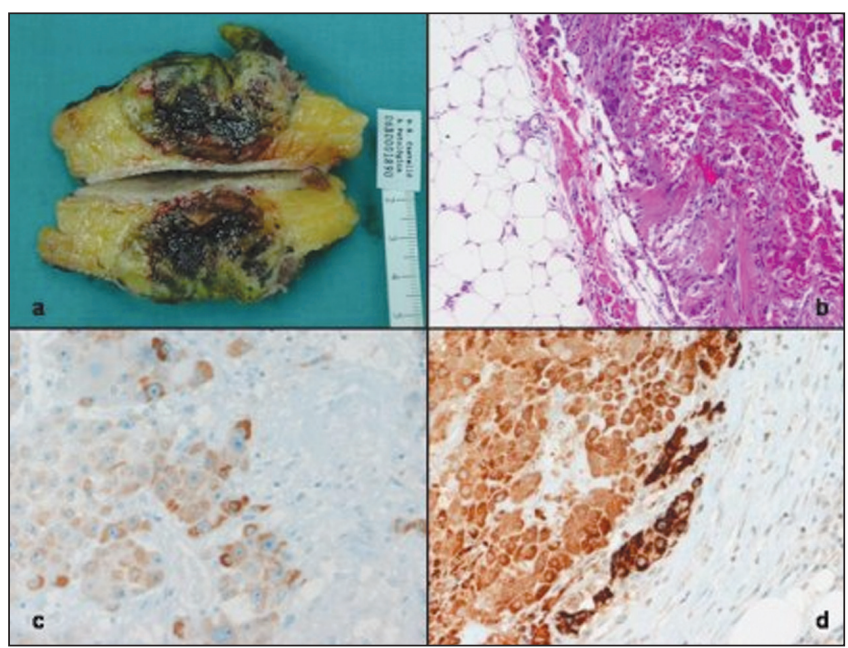

Fig. 3. Estudio histológico. a. Aspecto macroscópico de la lesión. b. Transición de tejido celular subcutáneo a tumoración (H-E, x10). c. Inmunorreactividad focal, de patrón granular, en el citoplasma de los hepatocitos para alfa-fetoproteína (alfa-fetoproteína, x20). d. Inmunorreactividad citoplasmática para el marcador específico de hepatocitos ("hepatocyte", x20).

se observó que el tumor estaba situado en el tejido subcutáneo adyacente a la dermis reticular, separado de este por una pseudocápsula fibrosa. Mostraba un patrón de crecimiento expansivo. Contenía haces y cordones de hepatocitos de diverso tamaño y morfología, con citoplasma intensamente eosinófilo, y núcleos hipercromáticos, de dimensiones variables, con moderado pleomorfismo (Fig. 3b). Se observaron, asimismo, algunas figuras de mitosis. Las células tumorales presentaron inmunorreactividad para alfa-fetoproteína (Fig. 3c) y marcador de he- 
patocitos ("hepatocyte") (Fig. 3d). El índice proliferativo (porcentaje de células positivas para Ki-67) fue elevado $(>20 \%)$. El aspecto histológico y la tinción inmunohistoquímica fueron prácticamente idénticos a los del tumor original.

Tras la cirugía, el postoperatorio fue satisfactorio y la enferma continúa en tratamiento mediante embolizaciones de su enfermedad hepática.

\section{DISCUSIÓN}

Se ha estimado que entre el 0,7 y el 9\% de los pacientes con tumores malignos, de cualquier origen, pueden llegar a desarrollar una metástasis cutánea o subcutánea (2). En la mujer, los tumores primarios se localizan, por orden de frecuencia, en la mama, en el colon y en la propia piel (melanoma), mientras que en los varones corresponden al pulmón, al colon y al melanoma (3). Las metástasis cutáneas o subcutáneas de los carcinomas hepatocelulares son todavía menos frecuentes, como consecuencia de la escasa tendencia a afectar dichos tejidos y de la menor incidencia de estos tumores primarios (2).

Se han comunicado diferentes mecanismos por los que un tumor hepático puede llegar a producir metástasis cutáneas, como son: el crecimiento en continuidad desde el parénquima hepático (4); la diseminación sistémica de la enfermedad (2) y la implantación o siembra tumoral tras la manipulación de la lesión por diferentes mecanismos tales como cirugía (5); inyección de etanol de forma terapéutica (6); o como en nuestro caso, tras la biopsia percutánea diagnóstica (7).

La punción aspiración con aguja fina ha demostrado su utilidad en el estudio de lesiones hepáticas, obteniendo, en la mayoría de ocasiones, una cantidad suficiente de tejido para llegar a un diagnóstico de certeza, con una sensibilidad y una especificidad cercanas al $100 \%(2,8)$. Por ello, esta prueba se ha convertido en una exploración habitual en la mayoría de centros hospitalarios. La PAAF puede llevarse a cabo guiada por ecografía o por TC y está especialmente indicada en las lesiones focales del hígado, aunque algunos autores han encontrado también buenos resultados en lesiones hepáticas difusas (2). A pesar de estas indudables ventajas, conviene recordar que, como sucedió en nuestro caso, esta prueba no está exenta de riesgo ya que, al igual que cualquier prueba invasiva, presenta cierta morbimortalidad. Así, en el estudio de Weiss y cols. (9) se demostró que, tras la realización de una PAAF sobre una lesión tumoral, el número de células en el trayecto de punción puede ser elevado, con valores cercanos a las 1.000 células malignas implantadas. A pesar de estas cifras tan alarmantes, Smith (10) evaluó más de 70.000 pacientes sometidos a punción aspiración con aguja fina en todo el territorio estadounidense, encontrando únicamente 4 casos con implantes tumorales en el trayecto de punción, aunque ninguno de ellos correspondía a biopsias hepáticas -2 fueron carcinomas renales, 1 cán- cer de páncreas y 1 cáncer cervical-. Por lo tanto, según este estudio, la frecuencia de implantación de células tumorales tras la PAAF es cercana al $0,006 \%$. Por otro lado, la mortalidad relacionada con este proceder es similar a la encontrada en pacientes sometidos a otras pruebas de imagen invasivas, con valores que oscilarían entre 0,018 y $0,096 \%(8,10)$.

Aunque de forma muy esporádica, diversos autores han comunicado casos similares al nuestro, con afectación cutánea o subcutánea por un carcinoma hepatocelular tras la biopsia hepática $(7,11,12)$. En algunas ocasiones, no puede determinarse con claridad si el origen de los implantes es realmente la PAAF u otro procedimiento diagnóstico/terapéutico en un mismo paciente, sin embargo, nuestro caso no ofrece dudas ya que, como se aprecia en la figura 2, la lesión metastásica se localizó, exactamente, en la zona de punción subxifoidea. La biopsia con tru-cut se extrajo envainada por el mismo trayecto de la PAAF, por lo que es menos probable que sea el origen del implante. Del mismo modo, la localización subcutánea de una única metástasis apoya nuestro diagnóstico.

Clínicamente, las metástasis cutáneas de los carcinomas hepatocelulares suelen manifestarse como nódulos angiomatosos, violáceos, de entre 1 a 2,5 cm de diámetro, de consistencia firme, indoloros y no ulcerados en superficie (2). Nuestro caso presentaba estas mismas características, aunque con un tamaño tumoral algo superior, lo que condicionó la aparición del dolor. Las lesiones cutáneas pueden presentar, asimismo, un aspecto similar a un granuloma piogénico (6), siendo únicamente distinguibles de este mediante el estudio anatomopatológico.

El estudio histológico e inmunohistoquímico de las metástasis cutáneas de tumores primarios internos puede ser difícil, especialmente en el caso de los tumores poco diferenciados. Por otro lado, puede ocurrir que tumores bien diferenciados -como muchos carcinomas-, al metastatizar, se conviertan en indiferenciados. Este fenómeno aparece frecuentemente en el caso de las metástasis cutáneas, y obliga a realizar estudios complementarios para determinar la estirpe celular del tumor (3). En lo que se refiere a las metástasis cutáneas de hepatocarcinoma, la aparición del anticuerpo monoclonal para las mitocondrias de hepatocitos ("OCHIE5-Hepatocyte-Hep Par l”) ha supuesto una importante ayuda para el diagnóstico de los tumores poco diferenciados. El caso que describimos, no obstante, presentaba características histológicas de hepatocarcinoma bien diferenciado, y resultó similar, tanto citológicamente como en el marcaje inmunohistoquímico, al tumor primario. Esto podría explicarse porque, al tratarse de una "siembra" tumoral directa del hepatocarcinoma primario, no habría lugar a una indiferenciación histológica del tumor.

Cuando se confirma el diagnóstico, el tratamiento de las metástasis cutáneas, siempre que sea posible por las condiciones del paciente y de la enfermedad hepática de base, debe ser la resección quirúrgica. 
En nuestra opinión, las complicaciones de la PAAF son tan esporádicas y con tan pocos casos publicados en la literatura médica -teniendo en cuenta el total de biopsias percutáneas con aguja fina que se realizan en todo el mundo-, que puede aceptarse que el riesgo de implantación es mínimo. Sin embargo, el caso presentado sirve para alertar de una complicación infrecuente pero que, dada su extrema gravedad, debe ser conocida y tenida en cuenta por todos aquellos profesionales involucrados en el proceso diagnóstico-terapéutico de estos pacientes.

\section{BIBLIOGRAFÍA}

1. Bravo AA, Sheth SG, Chopra S. Liver biopsy. N Engl J Med 2001; 344: 495-500.

2. Jegou J, Peruzzi P, Arav E, Pluot M, Jaussaud R, Remy G. Métastases mixtes cutáneo-osseuses révélatrices d'un carcinome hépatocellulaire. Gastroenterol Clin Biol 2004; 28: 804-6.

3. Kanitakis J, Causeret AS, Claudy A, Scoazec JY. Cutaneous metastasis of hepatocellular carcinoma diagnosed with hepatocyte paraffin (Hep Par 1) antibody immunohistochemistry. J Cutan Pathol 2003; 30: $637-40$
4. Knight TE, Woo AS, Blaisdell JM. Hepatocellular carcinoma invasive to chest wall. Int J Dermatol 1992; 31: 273-6.

5. Koffi E, Moutardier V, Sauvanet A, Noun R, Flejou JF, Belghiti J. Wound recurrence after resection of hepatocellular carcinoma. Liver Transpl Surg 1996; 2: 301-3.

6. Lee MC, Huang YL, Yang CH, Kuo TT, Hong HS. Cutaneous seeding of hepatocellular carcinoma due to percutaneous ethanol injection and masquerading as a pyogenic granuloma. Dermatol Surg 2004; 30: 438-40.

7. Navarro F, Taourel P, Michel J, Perney P, Fabre JM, Blanc F, et al. Diaphragmatic and subcutaneous seeding of hepatocellular carcinoma following fine-needle aspiration biopsy. Liver 1998; 18: 251-4.

8. Torzilli G, Minagawa M, Takayama T, Inoue K, Hui AM, Kubota K, et al. Accurate preoperative evaluation of liver mass lesions without fine-needle biopsy. Hepathology 1999; 30: 889-93.

9. Weiss L, Ward PM. Effects of metastasic cascades on metastasic patterns: studies on colon-26 carcinomas in mice. Int J Cancer 1998; 41: 450-5.

10. Smith E. Complications of percutaneous abdominal fine-needle biopsy. Review. Radiology 1991; 178: 253-8.

11. Isobe H, Imari Y, Sakai H, Sakamoto S, Nawata H. Subcutaneous seeding of hepatocellular carcinoma following fine-needle aspiration biopsy. J Clin Gastroenterol 1993; 17: 350-2.

12. Yamada N, Shinzawa H, Ukai K, Wakabayashi H, Togashi H, Takahashi T, et al. Subcutaneous seeding of small hepatocellular carcinoma after fine needle aspiration biopsy. J Gastroenterol Hepatol 1993; 8: $195-8$. 